\title{
Comparison of Morphine and Remifentanil on the Duration of Weaning from Mechanical Ventilation
}

\author{
Jae Myeong Lee, M.D., Seong Heon Lee, M.D. , Sang Hyun Kwak, M.D. , Hyeon Hui Kang, M.D. ', Sang Haak Lee, M.D. ${ }^{\dagger}$, \\ Jae Min Lim, M.D. ${ }^{\ddagger}$, Mi Ae Jeong, M.D. ${ }^{\S}$, Young Joo Lee, M.D." , and Chae Man Lim, M.D. ${ }^{`}$ \\ Department of Surgery, Ajou University School of Medicine, Suwon; *Department of Anesthesiology and Pain Medicine, Chonnam National University, Medical School, Gwangju; \\ ${ }^{\dagger}$ Division of Pulmonology, Critical Care and Sleep Medicine, Department of Internal Medicine, St. Paul's Hospital, The Catholic University of Korea, Seoul; " Department of Pulmonology, \\ Kangreung Asan Medical Center, Kangreung; \& Department of Anesthesiology and Pain Medicine, Hanyang University school of Medicine, Seoul; "Department of Anesthesiology and \\ Pain Medicine, Ajou University School of Medicine, Suwon; "University of Ulsan, College of Medicine, Asan Medical Center, Seoul, Korea
}

2014 November 29(4):281-287 / http://dx.doi.org/10.4266/kjccm.2014.29.4.281

We found an error in this article. The author's affiliation should be corrected as following:

from “Jae Myeong Lee, M.D., Seong Heon Lee, M.D.", Sang Hyun Kwak, M.D.", Hyeon Hui Kang, M.D.†, Sang Haak Lee, M.D. ${ }^{\dagger}$, Jae Min Lim, M.D. , Mi Ae Jeong, M.D. ${ }^{\S}$, Young Joo Lee, M.D.", and Chae Man Lim, M.D."

Department of Surgery, Ajou University School of Medicine, Suwon; "Department of Anesthesiology and Pain Medicine, Chonnam National University, Medical School, Gwangju; "Division of Pulmonology, Critical Care and Sleep Medicine, Department of Internal Medicine, St. Paul's Hospital, The Catholic University of Korea, Seoul; "Department of Pulmonology, Kangreung Asan Medical Center, Kangreung; ${ }^{\S}$ Department of Anesthesiology and Pain Medicine, Hanyang University school of Medicine, Seoul; "Department of Anesthesiology and Pain Medicine, Ajou University School of Medicine, Suwon; "University of Ulsan, College of Medicine, Asan Medical Center, Seoul, Korea"

to "Jae Myeong Lee, M.D. ${ }^{\text {, }}$, Seong Heon Lee, M.D. ${ }^{2}$, Sang Hyun Kwak, M.D. ${ }^{2}$, Hyeon Hui Kang, M.D. ${ }^{3}$, Sang Haak Lee, M.D. ${ }^{3}$, Jae Min Lim, M.D. ${ }^{4}$, Mi Ae Jeong, M.D. ${ }^{5}$, Young Joo Lee, M.D. ${ }^{6}$, and Chae Man Lim, M.D. ${ }^{7}$

${ }^{1}$ Department of Surgery, Ajou University School of Medicine, Suwon; ${ }^{2}$ Department of Anesthesiology and Pain Medicine, Chonnam National University, Medical School, Gwangju; ${ }^{3}$ Division of Pulmonology, Critical Care and Sleep Medicine, Department of Internal Medicine, St. Paul's Hospital, The Catholic University of Korea, Seoul; ${ }^{4}$ Department of Pulmonology, Gangreung Asan Medical Center, University of Ulsan College of Medicine, Gangreung; ${ }^{5}$ Department of Anesthesiology and Pain Medicine, Hanyang University school of Medicine, Seoul; ${ }^{6}$ Department of Anesthesiology and Pain Medicine, Ajou University School of Medicine, Suwon; ${ }^{7}$ Department of Pulmonary and Critical Care Medicine, Asan Medical Center, University of Ulsan College of Medicine, Seoul, Korea"

We apologize for any inconvenience that may have caused. 